\title{
Multi-photon microscopy in neuroscience
}

\author{
Winfried Denk, \\ Max-Planck Institute for Medical Research, Heidelberg, Germany, \\ denk@ mpimf-heidelberg.mpg.de
}

The study of the nervous system requires to an exceptional extent observation of and experimentation on intact tissue. There, in particular, high-resolution optical microscopy benefits from the inherent advantages of multi-photon fluorescence excitation. Several cases will be presented from a number of different tissues and organisms, where multi-photon excited laser scanning fluorescence microscopy has been an essential experimental tool. Those examples include the discovery of biochemical coincidence detection in synaptic spines and the clarification of the underlying mechanism; the observation of sensory evoked dendritic signaling in intact animals and the observation of light induced calcium signals in the intact retina. Recently a fiber coupled two-photon microscopy has been developed that allows the imaging in moving animals.

Optical microscopy is virtually the only technique capable to create high-resolution images on of living specimens. The most widely used class of optical microscopes makes use of fluorescent molecules, which can provide a very high signal to background ratio, molecular specificity, and, due to spectral sensitivity to the molecular environment the ability to measure chemical parameters with exquisite spatial resolution. In intact tissue optical sectioning and efficient use of excited fluorescence are essential. Conventional techniques don't permit the simultaneous fulfillment of these requirements, especially in the presence of scattering by the specimen. Wide-field microscopy using cooled CCDs or intensified video cameras efficiently detects fluorescence but lacks optical sectioning. Resolution and contrast in the wide-field image degrade quickly as scattering is introduced. Optical sectioning is provided by the confocal microscope but there fluorescence collection efficiency is poor even in clear specimens and becomes abysmal in scattering specimens. Multi-photon excitation microscopy (MPM) allows the combination of optical sectioning and collection efficiency even in the presence of severe scattering (Denk 1996; Denk and Svoboda 1997). A particularly convincing test of this ability is high-resolution functional imaging inside the intact brain of living rats (Svoboda, Denk et al. 1997; Helmchen, Svoboda et al. 1998; Svoboda, Helmchen et al. 1999). The questions addressed in those experiments concerned the antidromic propagation of action potentials into the dendritic tree of different types of principal cells in cortex. Recently it has been demonstrated that it should be possible to extend such studies to the alert brain with the help of a fiber coupled two-photon microscope that is miniaturized sufficiently to be mounted onto the head of a freely moving rodent (Helmchen, Fee et al. 2001).

If repeated measurements have to be preformed on small structures the reduction of photodamage and photobleaching that results from the efficient collection of fluorescent photons can be essential. This has been exploited in multi-photon microscopy studies of dendritic spines, which are in some sense the 'transistors' of the brain. Spines serve as chemical compartments (Svoboda, Tank et al. 1996) that allow each neuron to have tens of thousands of independent biochemical coincidence detectors. Coincidence detection in spines was first demonstrated convincingly using the multi-photon microscope (Yuste and Denk 1995). MPM studies were subsequently used to examine molecular mechanisms responsible for coincidence detection in hippocampal (Yuste, Majewska et al. 1999) and neocortical cells (Koester and Sakmann 1998). Recently a calcium based coincidence detection using an entirely different molecular mechanism was found in cerebellar Purkinje cells (Wang, Denk et al. 2000). Fluctuation analysis has been used to determine the number of ion channels in single spines (Sabatini and Svoboda 2000).

Another very fruitful area for the application of multi-photon microscopy is the retina, the highly light sensitive tissue in the back of the eye converting photons into the electrochemical signals used by the brain to convey and represent information. In the retina fluorescence microscopy, in particular, is inherently hampered by the rather high illumination intensities, which are needed for the sufficient excitation of fluorescence. Such high light fluxes quickly blind the retina by bleaching out the photo pigments that are needed for light detection. It has, therefore, been impossible to use fluorescence based indicators of 
biochemical signaling to detect biochemical signals arising in response to visual stimulation. The multiphoton microscope remedies the situation because the infrared light used for 2-photon excitation of xanthene-based biochemical indicators does not directly stimulate or bleach the photo-pigments. Out-offocus two-photon excitation of visual pigment can be neglected in most of the signal processing cell layers, which are distinct and sufficiently far from the photoreceptor layer (Denk and Detwiler 1999). Recently this technique was used to elucidate the neural computation necessary for the detection of image motion by the retina (Euler, Detwiler et al. 2002).

\section{References}

Denk, W. (1996). “Two-Photon Excitation in Functional Biological Imaging.” Journal of Biomedical Optics 1(3): 296-304.

Denk, W. and P. B. Detwiler (1999). "Optical recording of light-evoked calcium signals in the functionally intact retina." Proceedings of the National Academy of Sciences of the United States of America 96(12): 7035-7040.

Denk, W. and K. Svoboda (1997). "Photon upmanship: Why multiphoton imaging is more than a gimmick." Neuron 18(3): 351-357.

Euler, T., P. B. Detwiler and W. Denk (2002). "Directionally selective calcium signals in dendrites of starburst amacrine cells." Nature 418(6900): 845-52.

Helmchen, F., M. S. Fee, D. W. Tank and W. Denk (2001). “A miniature head-mounted two-photon microscope. high-resolution brain imaging in freely moving animals.” Neuron 31(6): 903-12.

Helmchen, F., K. Svoboda, W. Denk and D. W. Tank (1998). "Dendritic calcium dynamics in deep neocortical neurons measured in vivo using two-photon microscopy." Soc. Neurosci. Abstr. 24: 79.

Koester, H. J. and B. Sakmann (1998). "Calcium dynamics in single spines during coincident pre- and postsynaptic activity depend on relative timing of back-propagating action potentials and subthreshold excitatory postsynaptic potentials.” Proc Natl Acad Sci U S A 95(16): 9596-601.

Sabatini, B. L. and K. Svoboda (2000). "Analysis of calcium channels in single spines using optical fluctuation analysis." Nature 408(6812): 589-93.

Svoboda, K., W. Denk, D. Kleinfeld and D. Tank (1997). "In Vivo Dendritic Calcium Dynamics in Neocortical Pyramidal Neurons.” Nature 385(6612): 161-165.

Svoboda, K., F. Helmchen, W. Denk and D. W. Tank (1999). "Spread of excitation in Layer 2/3 pyramidal Neurons in Rat Barrel Cortex in vivo." Nature Neuroscience 2(1): 65-73.

Svoboda, K., D. W. Tank and W. Denk (1996). "Direct measurement of coupling between dendritic spines and shaft." Science 272(5262): 716-719.

Wang, S. S. H., W. Denk and M. Hausser (2000). "Coincidence detection in single dendritic spines mediated by calcium release." Nature Neuroscience 3(12): 1266-1273.

Yuste, R. and W. Denk (1995). "Dendritic spines as basic functional units of neuronal integration." Nature 375(6533): 682-684.

Yuste, R., A. Majewska, S. S. Cash and W. Denk (1999). "Mechanisms of calcium influx into hippocampal spines: Heterogeneity among spines, coincidence detection by NMDA receptors, and optical quantal analysis." Journal of Neuroscience 19(6): 1976-1987. 